\title{
On-Board Doppler Filtering for Data Volume Reduction in Spaceborne SAR Systems
}

\author{
Michelangelo Villano, Gerhard Krieger \\ Microwaves and Radar Institute \\ German Aerospace Center (DLR) \\ Wessling, Germany \\ michelangelo.villano@dlr.de,gerhard.krieger@dlr.de
}

\author{
Vincenzo Del Zoppo \\ Department of Physics and Astronomy \\ University of Catania \\ Catania, Italy \\ vinc.delzoppo@live.it
}

\begin{abstract}
High-resolution wide-swath (HRWS) synthetic aperture radar (SAR) systems based on digital beamforming (DBF) in elevation are very attractive for the observation of dynamic processes on the Earth's surface. However, HRWS systems are inherently associated with a huge data volume. Moreover, in order to comply with azimuth ambiguity requirements, a pulse repetition frequency (PRF) much higher than the required processed Doppler bandwidth is often desirable. The data volume can be drastically reduced, if onboard Doppler filtering and decimation are performed prior to downlink. A finite impulse response (FIR) filter with a relatively small number of taps suffices to completely suppress the additional ambiguous components and recover the original impulse response, provided that the filter's transfer function is compensated for in the processing. This strategy is also applicable to staggered-SAR systems, where on-board Doppler filtering and resampling can be jointly implemented.
\end{abstract}

Index Terms-Synthetic aperture radar (SAR), highresolution wide-swath (HRWS) SAR imaging, staggered SAR, data volume, on-board Doppler filtering.

\section{INTRODUCTION}

Synthetic aperture radar (SAR) is a remote sensing technique, capable of providing high-resolution images independent of weather conditions and sunlight illumination. This makes SAR very attractive for the systematic observation of dynamic processes on the Earth's surface. However, conventional SAR systems are limited, in that a wide swath can only be achieved at the expense of a degraded azimuth resolution [1]. This limitation can be overcome by highresolution wide-swath (HRWS) systems based on digital beamforming (DFB) in elevation, where multiple swaths can be simultaneously imaged using multiple receive beams [2]. Moreover, if the pulse repetition interval (PRI) is continuously varied (staggered SAR), it is also possible to get rid of the "blind ranges", present between adjacent swaths, as the radar cannot receive while it is transmitting [3], [4]

Due to their resolution and coverage requirements, however, HRWS systems are inherently associated with a huge data volume, thereby increasing the demands for internal data storage, downlink, ground processing and archiving. Moreover, in order to comply with azimuth ambiguity requirements, a pulse repetition frequency $P R F$ much higher than the required processed Doppler bandwidth $B_{p}$ is often desirable. This determines a further increase of the data volume to be downlinked with a direct impact on the cost of the mission. As an example, for a SAR system characterized by a pulse repetition frequency $P R F=1492 \mathrm{~Hz}$ and a processed Doppler bandwidth $P B W=600 \mathrm{~Hz}$, due to the azimuth oversampling, i.e., the use of a PRF larger than the processed Doppler bandwidth, the data volume to be downlinked increases by almost $150 \%$. The system, in fact, downlinks data included in the Doppler frequency interval [-PRF/2, $P R F / 2]$, while only data in the Doppler frequency interval $[-P B W / 2, P B W / 2]$ are needed to achieve the desired azimuth resolution. The information contained in the Doppler frequency intervals [$P R F / 2,-P B W / 2]$ and $[P B W / 2, P R F / 2]$ is useless and discarded in the SAR processing.

\section{DATA VOLUME REDUCTION STRATEGY}

If data were just decimated (e.g., by a factor of 2 in the considered example) prior to downlink, a considerable degradation of the azimuth-ambiguity to signal ratio (AASR) would occur. Fig. 1 (a) shows the power spectral density of the azimuth SAR signal for a planar antenna of length $L=10 \mathrm{~m}$ and a wavelength $\lambda=0.2384 \mathrm{~m}$ (L-band). The unambiguous energy, the ambiguous energy and the additional ambiguous energy due to the decimation are highlighted in green, red, and blue, respectively. As is apparent, the additional ambiguous energy due to decimation is significant. However, if Doppler low-pass filtering is performed before decimation, the additional ambiguous energy due to decimation can be substantially reduced, as shown in Fig. 1 (b).

Due to the large amount of data, acquired by typical HRWS systems, the number of on-board operations per sample has to be minimized, while avoiding a degradation of the impulse response. The Doppler low-pass filtering can be performed in time domain using a finite impulse response (FIR) filter with a relatively small number of taps. The filter will introduce a distortion of the Doppler spectrum of the signal, which can be compensated for in the SAR processing (on ground).

This work only analyzes the case of decimation by an integer factor, as this is associated with a straightforward implementation and a much lower computational cost, but this strategy can be also used in case of rational decimation factor.

Fig. 2 shows a block diagram of the overall data volume reduction strategy. 


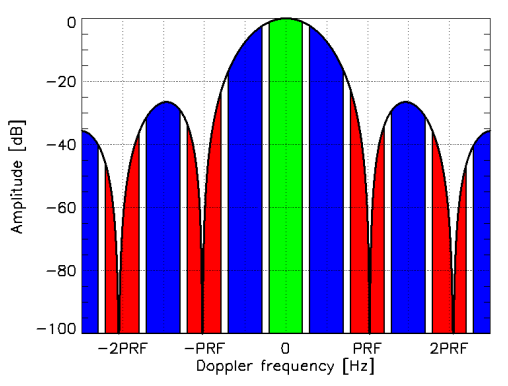

(a)

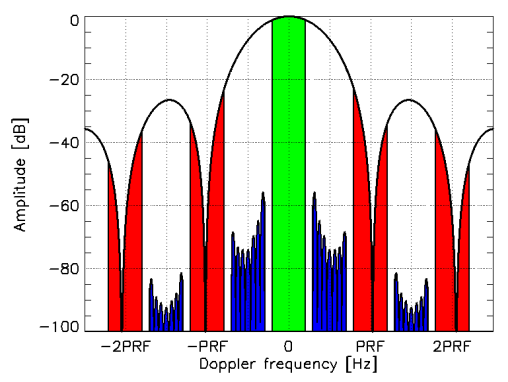

(b)

Figure 1. Power density spectrum of the azimuth SAR signal. The energy of the unambiguous component, the ambiguous components, and the additional ambiguous components due to decimation are highlighted in green, red and blue, respectively. (a) Only decimation (no low-pass filtering). (b) Low-pass filtering and decimation.

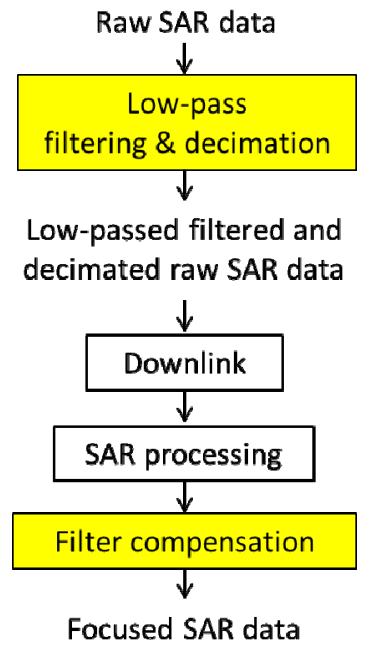

Figure 2. Block diagram of the proposed data volume reduction strategy.

\section{LOW-PASS FILTER DESIGN}

Different criteria for the design of the FIR low-pass filter are considered in the following, namely the FIR filter design by windowing, the FIR Wiener filter and a method based on the minimum variance distortionless response (MVDR) or Capon beamformer.

\section{A. FIR Filter Design by Windowing}

According to this criterion, the impulse response $h[n]$ of the FIR low-pass filter is obtained by windowing the impulse response of the ideal low-pass filter $h_{d}[n][5]$

$$
h[n]=h_{d}[n] w[n]
$$

where

$$
h_{d}[n]=\sin \left(\omega_{C} n\right) / \pi n
$$

and the cut-off radian frequency $\omega_{C}$ is given by

$$
\omega_{C}=\pi P B W / P R F
$$

Common windows $w[n]$ are considered, namely rectangular, Bartlett, Hanning, Hamming, and Blackman, defined as in [5]. Please consider that, according to this definition, $w[0]$ and $w[M-1]$ are equal to 0 for the Bartlett, Hanning and Blackman windows, therefore the effective number of taps for these three windows is reduced by 2 .

The transfer functions $H(f)$ of the filters, obtained by using the five aforementioned windows and the values of $P R F$ and $B_{p}$ of the considered example $\left(\omega_{C}=0.4 \pi\right)$, are represented in Fig. 3 for $M=25$. On the abscissa of the plot of Fig. 3 the Doppler frequencies $P B W / 2$ and $P R F / 2-P B W / 2$ are marked. A filter characterized by low values of $H(f)$ in the Doppler frequency interval $[P R F / 2-P B W / 2, P R F / 2]$ will provide a good suppression of the additional ambiguous components introduced by the decimation, while the values of the $H(f)$ in the interval $[P B W / 2, P R F / 2-P B W / 2]$ are not relevant, as these frequency components are cancelled within the SAR processing.

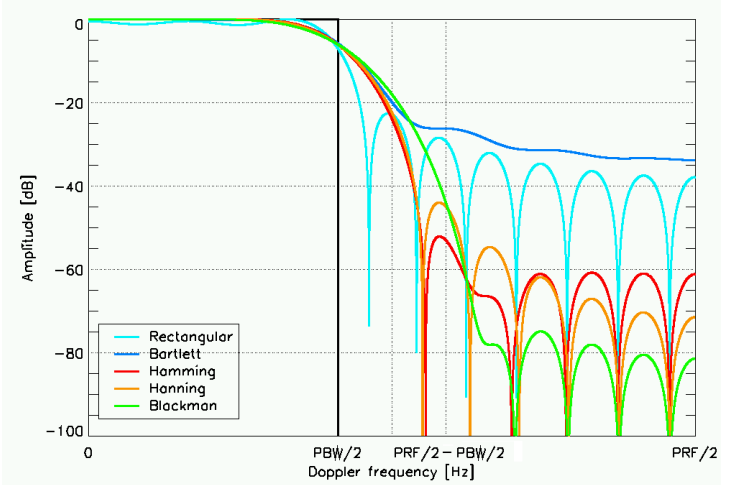

Figure 3. Transfer functions of the low-pass filter for $\omega_{C}=0.4 \pi$, different windows and $M=25$ taps. The transfer function of the ideal low-pass filter is shown in black.

\section{B. FIR Wiener Filter}

As an alternative to the use of common windows, the FIR filter could be also designed as a Wiener filter, i.e., exploiting the knowledge of the power spectral density (PSD) of the 
useful and disturbance signals [6]. In this case, the disturbance signal is given by the frequency components, which fold back to the main part of the spectrum after decimation. The coefficients of the FIR Wiener filter are given by

$$
\mathbf{h}=\mathbf{R}_{\mathrm{s}}^{-1} \mathbf{r}_{\mathbf{u}}
$$

where $\mathbf{R}_{\mathbf{s}}$ is the correlation matrix of the overall signal and $\mathbf{r}_{\mathbf{u}}$ is the correlation vector of the useful signal, given by

$$
\mathbf{R}_{\mathrm{s}}=\left[\begin{array}{cccc}
R_{s}[0] & R_{s}[-1] & \cdots & R_{s}[1-M] \\
R_{s}[1] & R_{s}[0] & \cdots & R_{s}[2-M] \\
\vdots & \vdots & \ddots & \vdots \\
R_{s}[M-1] & R_{s}[M-2] & \cdots & R_{s}[0]
\end{array}\right]
$$

and

$$
\mathbf{r}_{\mathbf{u}}=\left[\begin{array}{c}
R_{u}\left[-\frac{M-1}{2}\right] \\
\vdots \\
R_{u}[0] \\
\vdots \\
R_{u}\left[\frac{M-1}{2}\right]
\end{array}\right]
$$

respectively. $R_{s}[n]$ and $R_{u}[n]$ are related to the two way power pattern in azimuth $G^{2}(f)$ through the following relation:

$$
\begin{aligned}
R_{s}[n]= & R_{u}[n]+R_{d}[n]=\frac{1}{\pi} \int_{0}^{\omega_{C}} G^{2}\left(\omega=\frac{2 \pi f}{P R F}\right) \cos (\omega n) d \omega \\
& +\frac{1}{\pi} \int_{\pi-\omega_{C}}^{\pi} G^{2}\left(\omega=\frac{2 \pi f}{P R F}\right) \cos (\omega n) d \omega
\end{aligned}
$$

where a symmetric antenna azimuth pattern has been assumed and back-folded high-frequency components have been ignored.

Fig. 4 shows the transfer function of the 25-tap FIR Wiener filter obtained for $P R F=1492 \mathrm{~Hz}$ and $B_{p}=600 \mathrm{~Hz}$, assuming a uniformly-illuminated aperture of length $L=10 \mathrm{~m}$ and a satellite velocity $v_{S}=7473 \mathrm{~m} / \mathrm{s}$.

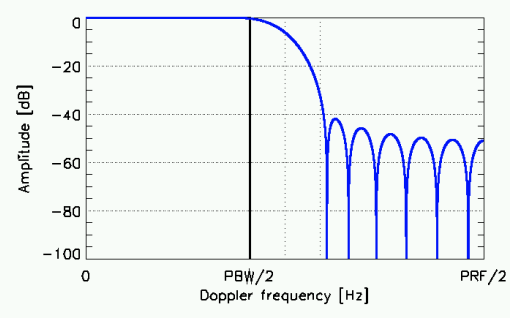

Figure 4. Transfer function of the Wiener filter for $\omega_{C}=0.4 \pi$, a uniformlyilluminated antenna of length $L=10 \mathrm{~m}$, and $M=25$ taps.

\section{Minimum Variance Distortionless Response (MVDR) or Capon Beamformer}

A further alternative to design the FIR Wiener filter is given by the MVDR or Capon beamformer [7]. The coefficients of the MVDR Wiener filter are given by

$$
\mathbf{h}=\mathbf{R}_{\mathbf{d}}^{-1} \mathbf{1}
$$

where $\mathbf{R}_{\mathbf{d}}$ is the correlation matrix of the disturbance signal, given by

$$
\mathbf{R}_{\mathbf{d}}=\left[\begin{array}{cccc}
R_{d}[0] & R_{d}[-1] & \cdots & R_{d}[1-M] \\
R_{d}[1] & R_{d}[0] & \cdots & R_{d}[2-M] \\
\vdots & \vdots & \ddots & \vdots \\
R_{d}[M-1] & R_{d}[M-2] & \cdots & R_{d}[0]
\end{array}\right]
$$

and $\mathbf{1}$ is a steering vector, whose components are all ones. The relationship between $R_{d}[n]$ and the two way antenna pattern $G(\omega=2 \pi f / P R F)$ is given in (7).

Fig. 5 shows the transfer function of the 9-tap MVDR filter obtained for $P R F=1492 \mathrm{~Hz}$ and $B_{p}=600 \mathrm{~Hz}$, assuming a uniformly illuminated aperture of length $L=10 \mathrm{~m}$ and a satellite velocity $v_{S}=7473 \mathrm{~m} / \mathrm{s}$.

While the Wiener filter (cf. Fig. 4) is characterized by a flat response in the Doppler frequency interval [0, $P B W / 2]$ and requires more taps (i.e., 25 in the example of Fig. 4 ) to provide a sufficient attenuation in the interval $[P R F / 2-P B W / 2, P R F / 2]$, the MVDR achieves a very good suppression of the higher frequencies with a much smaller number of taps (i.e., 9 in the example of Fig. 5). As a drawback, the MVDR filter introduces a significant attenuation in the interval $[0, P B W / 2]$ as well (up to $20 \mathrm{~dB}$ in the example of Fig. 5), which can be however compensated in the processing.

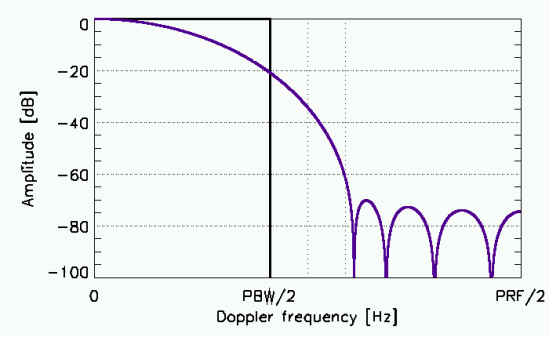

Figure 5. Transfer function of the MVDR filter for $\omega_{C}=0.4 \pi$, a uniformlyilluminated antenna of length $L=10 \mathrm{~m}$, and $M=9$ taps.

\section{PERFORMANCE ANALYSIS}

The performance of a system, where the described data volume reduction strategy is implemented, is evaluated and compared with a reference system, where the data volume reduction strategy is not applied, i.e., all data are downlinked. 


\section{A. 1-D Simulation-Constant PRF}

At first, a one-dimensional simulation with a uniformlyilluminated aperture is carried out. The relevant parameters of the SAR system are provided in Table 1.

Fig. 6 shows the azimuth impulse responses for the reference case (no data volume reduction) [Fig. 6 (a)] and for a reduction of the data volume by a factor of 2 , where different filters have been used [Fig. 6 (b)-(d)].

The azimuth resolution and the azimuth peak-to-side lobe ratio (PSLR), are equal to $13 \mathrm{~m}$ and $31.3 \mathrm{~dB}$, respectively, for the reference case, and remain unchanged for all filters, provided that the distortion of the Doppler spectrum of the signal, introduced by the low-pass filtering, is compensated for in the processing. This is done by multiplying the azimuth spectrum of the processed data by $\mathrm{C}(f)$, where

$$
C(f)= \begin{cases}\frac{1}{H(\omega=2 \pi f / P R F)} & ,|f| \leq P B W / 2 \\ 0 & , P B W / 2 \leq|f| \leq P R F / 2\end{cases}
$$

As far as azimuth ambiguities are concerned, it can be noticed that additional ambiguous peaks arise at $\pm 11.5 \mathrm{~km}$ in the azimuth impulse response of Fig. 6 (b). This means that the low-pass filter does not provide a sufficient suppression of the frequency components, which fold back after decimation. In all other cases the level of the aforementioned additional peaks is lower than $-60 \mathrm{~dB}$, therefore negligible.

In particular, the azimuth ambiguity-to-signal ratio (AASR) for a system with the described data volume reduction strategy for an integer decimation factor $p$ and assuming that $P R F \geq$ $p P B W$, can be analytically expressed as

$$
A A S R=\frac{\sum_{k=-\infty}^{k=\infty} \int_{k=0}^{P B W W / 2} \int^{P B W / 2} G^{2}(f+k P R F) Q^{2}(f) d f}{\int_{-P B W / 2}^{P B W / 2} G^{2}(f) Q^{2}(f) d f}
$$

$$
+\frac{\sum_{k=-\infty}^{k=\infty} \sum_{h=1}^{p-1} \int_{-P B W / 2}^{P B W / 2} G^{2}\left(f+\left(k+\frac{h}{p}\right) P R F\right) Q^{2}(f) \frac{H^{2}\left(f+\frac{h}{p} P R F\right)}{H^{2}(f)} d f}{\int_{-P B W / 2}^{P B W / 2} G^{2}(f) Q^{2}(f) d f}
$$

where $Q(f)$ accounts for the amplitude weighting of the Doppler spectrum applied in the processing (e.g. Hamming window and compensation of the azimuth antenna pattern), not including the compensation of the low-pass filter. The AASR is composed of two terms, where the first term is the AASR for a system, where no data volume reduction is performed ( -35.2 $\mathrm{dB}$ in the considered example), while the second one represents the AASR degradation due to the on-board filtering.
TABLE I RELEVANT SYSTEM PARAMETERS FOR THE 1-D SIMULATION

\begin{tabular}{|l|c|}
\hline \multicolumn{1}{|c|}{ Parameter } & Value \\
\hline Wavelength & $0.2384 \mathrm{~m}$ \\
\hline Orbit height & $770 \mathrm{~km}$ \\
\hline Closest slant range approach & $1000 \mathrm{~km}$ \\
\hline Antenna length & $10 \mathrm{~m}$ \\
\hline PRF & $1492 \mathrm{~Hz}$ \\
\hline Processed Doppler bandwidth & $600 \mathrm{~Hz}$ \\
\hline Decimation factor & 2 \\
\hline An azm procssing wing
\end{tabular}

An azimuth processing window is applied, which includes a Hamming weighting and a compensation of the azimuth antenna pattern and the low-pass filter.

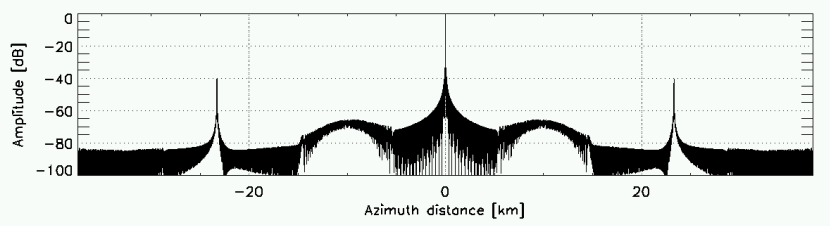

(a)

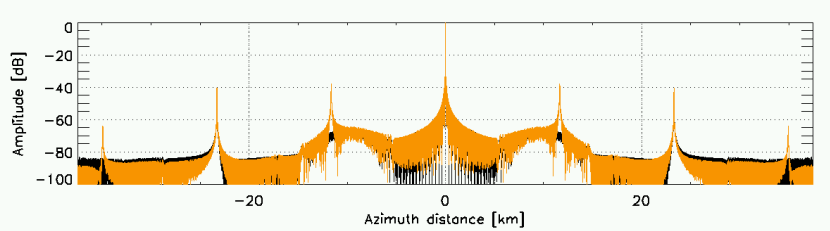

(b)

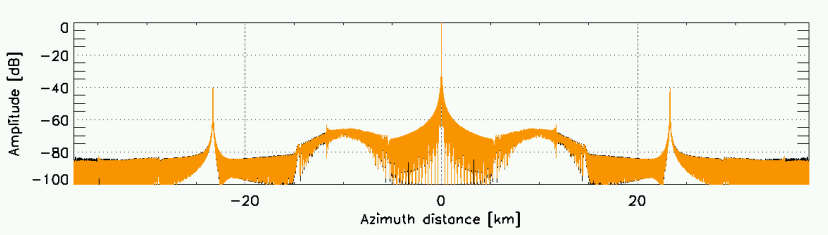

(c)

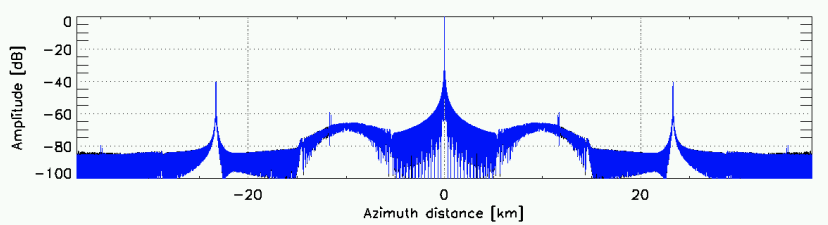

(d)

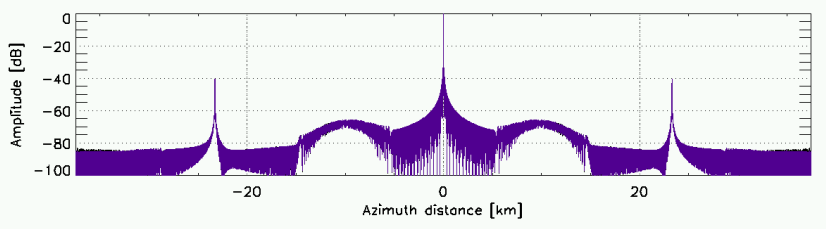

(e)

Figure 6. Azimuth impulse responses. (a) Reference case (no data volume reduction). (b) Hanning filter with $M=9$. (c) Hanning filter with $M=17$. (d) Wiener filter with $M=25$. (e) MVDR filter with $M=9$. 
Fig. 7 displays the AASR, evaluated using (11), as a function of the number of taps $M$, for the aforementioned filters and the system parameters of Table 1. As is apparent, for most of the filters, a relative small number of taps $(M<20$ for Hamming, Hanning, Blackman and Wiener filters and $M<10$ for the MVDR filter) suffices to make the AASR degradation due to the on-board filtering negligible.

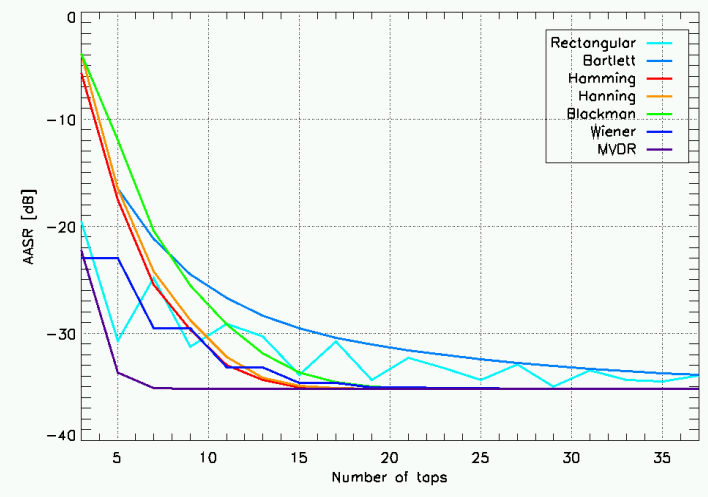

Figure 7. AASR $[\mathrm{dB}]$ as a function of the number of taps for the considered example and different filters.

Furthermore, it has to be remarked that no scaling of the signal-to-noise ratio (SNR) is associated with the proposed data volume reduction strategy, as also apparent from simulations.

Although the transfer function of the filter is compensated for in the SAR processing (cf. Fig. 2), an increase of the noise level is however foreseen for some of the filters, if the signal is further quantized prior to downlink. The quantization noise, in fact, is amplified, when compensating for the transfer function of the filter within the processing. Filter characterized by a flat response in the Doppler frequency interval [0, $P B W / 2]$, such as the presented Wiener filter (cf. Fig. 4), are robust to this problem, while other filters, such as the MVDR filter of Fig. 5, may lead to a severe increase of the noise level. In a context of data volume reduction, data are likely to be quantized, therefore it is worth to use a filter with a slightly higher number of taps, but robust to the amplification of quantization noise. This issue is further analyzed in [8], where raw TerraSAR-X data have been used and quantitative results are provided.

\section{B. 2-D Simulation - Constant PRF}

A two-dimensional simulation for a HRWS SAR system, which covers a $350-\mathrm{km}$ ground swath in quad polarization mode with 13 m azimuth resolution, using digital beamforming (DBF) on receive, has been furthermore performed. In this case a reflector antenna with multiple feeds has been considered. The relevant system parameters are provided in Table 2 .

Fig. 8 shows the AASR as a function of ground range for the reference case (no data volume reduction) and for a reduction of the data volume by a factor of 2, where a 25-tap Wiener coefficient has been used. As is apparent, the degradation is negligible. The 2D impulse responses for the two cases are shown in Fig. 9. The additional ambiguous peaks (absolute level of $-80 \mathrm{~dB}$ ) can be barely noticed comparing Fig. 9 (a) and Fig. 9 (b).
TABLE II.

RELEVANT SYSTEM PARAMETERS FOR THE 2-D SIMULATION

\begin{tabular}{|l|c|}
\hline \multicolumn{1}{|c|}{ Parameter } & Value \\
\hline Wavelength & $0.2384 \mathrm{~m}$ \\
\hline Orbit height & $770 \mathrm{~km}$ \\
\hline Incidence angle & $26.3^{\circ}-46.3^{\circ}$ \\
\hline Chirp bandwidth & $20 \mathrm{MHz}$ \\
\hline Range sampling frequency & $22 \mathrm{MHz}$ \\
\hline PRF & $1492 \mathrm{~Hz}$ \\
\hline Processed Doppler bandwidth & $600 \mathrm{~Hz}$ \\
\hline Duty cycle & $4 \%$ \\
\hline Tilt & $31.9^{\circ}$ \\
\hline Reflector diameter & $15 \mathrm{~m}$ \\
\hline RX excitation & 3 element uniform \\
\hline Decimation factor & \multicolumn{1}{c|}{2} \\
\hline $\begin{array}{l}\text { Range and azimuth Hamming } \\
\text { compensation of the azimuth antenna pattern and the low-pass filter, are } \\
\text { applied within the processing. }\end{array}$ & \begin{tabular}{c}
$(\alpha=0.6)$ as well as a \\
\hline
\end{tabular} \\
\hline
\end{tabular}

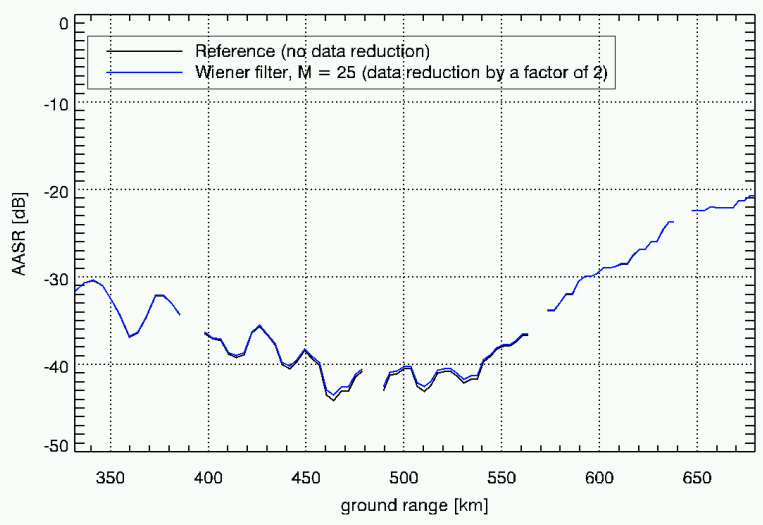

Figure 8. AASR [dB] vs. ground range for the constant PRF 2-D simulation.

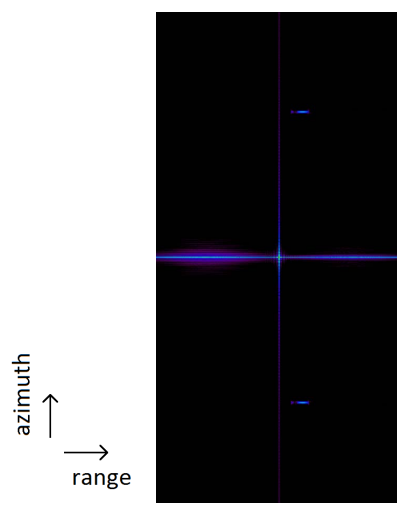

(a)

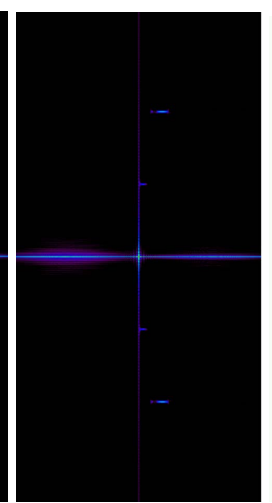

(b)
Figure 9. 2D impulse responses (constant PRF). (a) Reference case (no data volume reduction). (b) Data volume reduction by a factor of 2 using a 25-tap Wiener filter. 


\section{2-D Simulation-Staggered SAR}

If a constant PRF is used in combination with DBF on receive, blind ranges are present across the swath. A wide continuous swath can be achieved using staggered SAR, i.e. a continuous change of the PRI, in combination with DBF on receive [3], [4]. The proposed data volume reduction strategy can be applied to staggered SAR systems as well. In particular, the resampling of the non-uniform signal to a uniform grid and the low pass-filtering can be jointly implemented, thereby requiring a limited number of on-board operations. Moreover, for a staggered-SAR system, a rational decimation factor can be selected without a significant increase of the computational cost, just by properly choosing the resampling frequency.

A two-dimensional simulation has been performed for staggered SAR as well, using the same parameters given in Table 2 with a mean PRI on transmit equal to the reciprocal of the PRF of Table 2. Fig. 10 shows the integrated side-lobe-ratio (ISLR) as a function of ground range for the reference case (no data volume reduction) and for a reduction of the data volume by a factor of 2, where a 17-tap Wiener coefficient has been used. As is apparent, the degradation is negligible. The 2D impulse responses for the two cases are shown in Fig. 11, where no differences can be noticed.

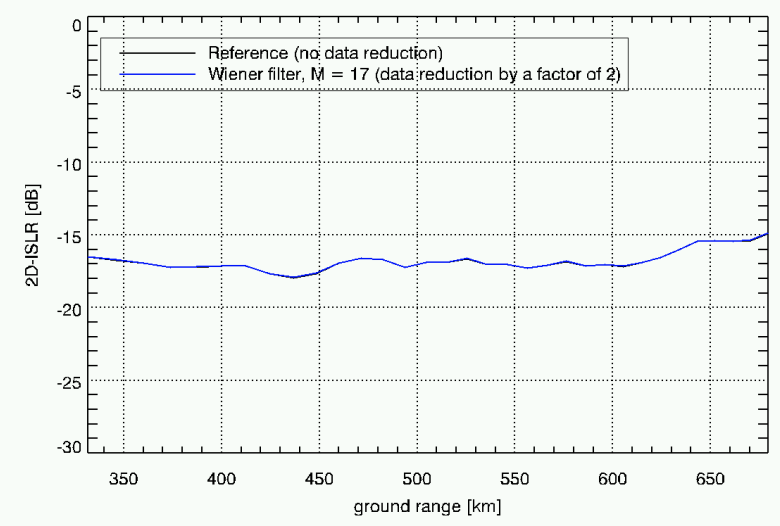

Figure 10. 2D-ISLR [dB] vs. ground range for the staggered SAR 2-D simulation.

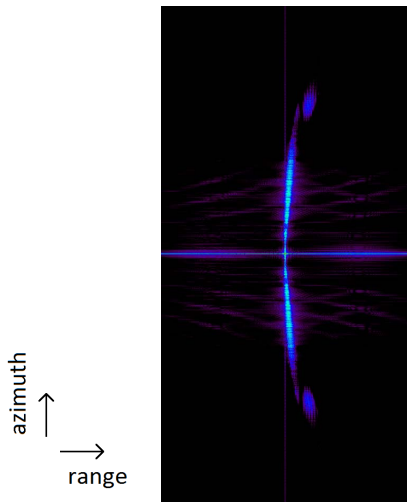

(a)

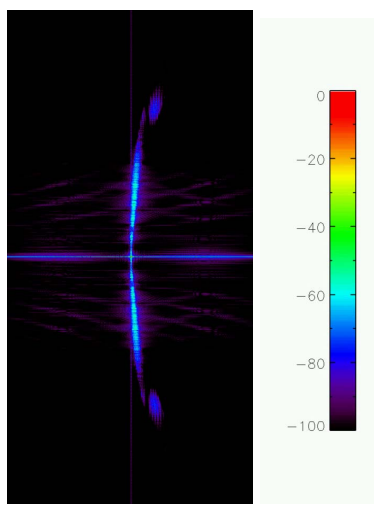

(b)
Figure 11. 2D impulse responses (staggered SAR). (a) Reference case (no data volume reduction). (b) Data volume reduction by a factor of 2 using a 17tap Wiener filter.

\section{CONCLUSIONS AND OUTLOOK}

A strategy for data volume reduction in spaceborne SAR system has been presented, where on-board Doppler filtering and decimation are performed on raw data prior to downlink. Different criteria for the design of the FIR filter have been proposed. It has been furthermore shown that a filter with a relatively small number of taps suffices to completely suppress the additional ambiguous components and recover the original impulse response, provided that the filter's transfer function is compensated for in the processing. This strategy is also applicable to staggered-SAR systems, where on-board Doppler filtering and resampling can be jointly implemented.

Although further analyses with simulations and real raw data are needed to better assess the performance for different scenarios and the implementation of the technique for rational decimation factor has to be better defined, from the first results the proposed strategy already shows a great potential for data volume reduction and should be therefore considered for the design of future spaceborne SAR systems.

\section{REFERENCES}

[1] J. C. Curlander and R. N. McDonough, "Synthetic Aperture Radar: Systems and Signal Processing," New York: Wiley, 1991.

[2] G. Krieger, N. Gebert, M. Younis, F. Bordoni, A. Patyuchenko, and A. Moreira, "Advanced concepts for ultra-wide-swath SAR imaging," Proceedings of EUSAR, Friedrichshafen, Germany, 2008.

[3] M. Villano, G. Krieger, and A. Moreira, "Staggered SAR: high resolution wide-swath imaging by continuous PRI variation," IEEE Trans. Geosci. Remote Sens., in press.

[4] M. Villano, G. Krieger, and A. Moreira, "A novel processing strategy for staggered SAR," IEEE Geosci. Remote Sens. Lett., in press.

[5] A.V. Oppenheim, R.W. Schafer, J.R.Buck, "Discrete-Time Signal Processing. Upper Saddle River,” NJ, USA: Prentice Hall, 1999.

[6] S. M. Kay, "Fundamentals of Statistical Signal Processing, vol. 1, Upper Saddle River," NJ, USA: Prentice-Hall, 1993.

[7] J. Capon, "High resolution frequency-wavenumber spectrum analysis," Proc. IEEE, vol. 57, pp. 1408-1418, Aug. 1969.

[8] M. Villano, M. Martone, V. Del Zoppo, B. Bräutigam, and G. Krieger, "Joint effects of on-board Doppler filtering and quantization in spaceborne SAR systems," IEEE GOLD Remote Sensing Conference, Berlin, Germany, 2014, unpublished. 